This item was submitted to Loughborough's Research Repository by the author.

Items in Figshare are protected by copyright, with all rights reserved, unless otherwise indicated.

\title{
Joint parameter optimization for perpetual nanonetworks and maximum network capacity
}

PLEASE CITE THE PUBLISHED VERSION

http://dx.doi.org/10.1109/TMBMC.2016.2564967

PUBLISHER

(C) IEEE

VERSION

AM (Accepted Manuscript)

LICENCE

CC BY-NC-ND 4.0

\section{REPOSITORY RECORD}

Yao, Xin-Wei, Wan-Liang Wang, and Shuang-Hua Yang. 2019. "Joint Parameter Optimization for Perpetual Nanonetworks and Maximum Network Capacity”. figshare. https://hdl.handle.net/2134/22531. 


\title{
Joint Parameter Optimization for Perpetual Nanonetworks and Maximum Network Capacity
}

\author{
Xin-Wei Yao, Wan-Liang Wang, and Shuang-Hua Yang, Senior Member, IEEE
}

\begin{abstract}
One of the major bottlenecks in nanonetworks is the very limited energy that can be accessed by nanodevices. To achieve perpetual data transmission, it is required to investigate in-depth the relationship between energy harvesting and consumption, and the underlying constraints in nanonetworks. In this paper, the tradeoff between energy harvesting and consumption is analyzed by considering the peculiarities of $\mathrm{THz}$ communication. First, based on the TS-OOK scheme and constrained energy in nanodevices, the upper bound of the transmitted pulse amplitude is presented. Second, given the proposed mathematical expression of the signal-to-interferencenoise ratio (SINR) in multi-user nanonetworks, the lower bound of pulse amplitude is presented to satisfy the required SINR threshold. Third, the minimum spreading factor is derived to guarantee the perpetual nanonetworks by considering the energy harvesting-consumption tradeoff. Finally, the maximization of network capacity is investigated by jointly optimizing the parameters of spreading factor, transmission distance, amplitude of the transmitted pulse, pulse probability, and node density for perpetual nanonetworks. The simulation results demonstrate short transmission distance and small spreading factor are recommended to improve the network capacity. Moreover, pulse probability, pulse amplitude, spreading factor, and node density are required to be comprehensively manipulated to achieve the maximum network capacity and perpetual communication.
\end{abstract}

Index Terms-Energy consumption, energy harvesting, perpetual nanonetworks, network capacity, THz band.

\section{INTRODUCTION}

$\mathbf{N}$ ANOTECHNOLOGY is enabling the development of novel devices in the order of a few cubic micrometers in size, which can accomplish only very simple tasks [1], [2]. Due to the very limited abilities of individual nanodevice, communication among nanodevices will expand the potential applications of single nanodevice through collaboration.

Manuscript received April 25, 2015; revised October 16, 2015 and March 10, 2016; accepted April 1, 2016. This work was supported in part by the National Natural Science Foundation of China under Grant 61379123 and Grant 61402414, in part by the Natural Science Foundation of Zhejiang Province, China, under Grant LQ14F020005 and Grant LQ15E050006, in part by the Public Project of Science Technology Department of Zhejiang Province under Grant 2015C31007, and in part by the Research Program of Educational Commission of Zhejiang Province of China under Grant Y201431815. The associate editor coordinating the review of this paper and approving it for publication was A. Eckford.

X.-W. Yao and W.-L. Wang are with the College of Computer Science and Technology, Zhejiang University of Technology, Hangzhou 310023, China (e-mail: xwyao@zjut.edu.cn; wwl@zjut.edu.cn).

S.-H. Yang is with the Department of Computer Science, Loughborough University, Loughborough LE11 3TU, U.K. (e-mail: s.h.yang@lboro.ac.uk).

Digital Object Identifier 10.1109/TMBMC.2016.2564967
The resulting nanonetworks will be able to boost the range of applications of nanotechnology in biomedical, environmental and military fields, such as intra-body health monitoring systems, distributed air pollution control, and nanosensor networks [3]. Two main alternatives for communication in the nanoscale have been considered [1], named molecular communication [4]-[6] and electromagnetic communication [2]. In detail, molecular communication is defined as the transmission and reception of information encoded in molecules, while electromagnetic communication is defined as the transmission and reception of electromagnetic radiation from nanodevices using novel nanomaterials [1]. In this paper, we focus on electromagnetic communication among nanodevices.

According to the limited size of nanodevices, scaling a metallic antenna down to a few hundred nanometers would impose the use of very high operating frequencies, thus, drastically limiting the communication range of nanodevices. Alternatively, graphene [7] and its derivatives [8], such as Carbon Nanotubes [9] and Graphene Nanoribbons [10], can be used to develop nanoantennas able to radiate at much lower frequencies. This frequency band, named $\mathrm{THz}$ Band, spans the frequency range from $0.1 \mathrm{THz}$ to $10.0 \mathrm{THz}$ [8]. For the time being, due to the strict constraints of a single nanodevice in terms of size and energy, no integrated technology has been presented to generate a high power carrier frequency in the $\mathrm{THz}$ Band. As a result, the traditional wireless communication mechanisms on the basis of continuous transmission signals may be not suitable for nanonetworks with limited hardware. Inspired by the huge bandwidth provided by the THz Band [11], new pulse-based communication mechanisms have been envisioned as the candidates for nanonetworks by the exchange of a few femtoseconds long pulses [12]-[14]. Moreover, the energy requirement on the transmission is relaxed by distributing short pulses over the time rather than continuous signals.

On the other hand, the very limited energy stored in the nanobatteries is another major challenge in nanonetworks. Therefore, energy harvesting system has been considered to provide energy for nanodevices. Unfortunately, conventional energy harvesting systems, such as solar energy, or wind power, cannot be adopted owing to the limited size of nanodevices, just several cubic micrometers. Recently, piezoelectric nanogenerators have been proposed to recharge the nanodevices [15], [20]-[22]. In addition, some works considered the energy consumption process of electromagnetic communication in the THz Band, and even for multi-hop nanonetworks in the aspect of MAC-layer or Network-layer [27], [28]. 


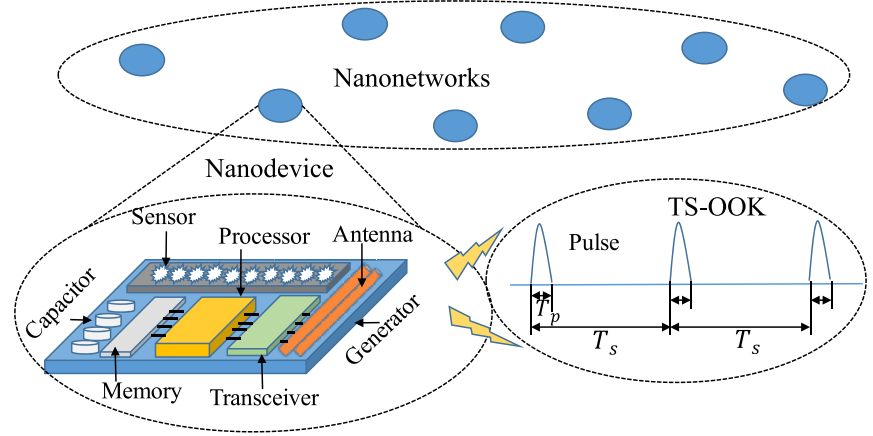

Fig. 1. Structure of a simplified nanonetwork.

However, these works do not consider the integrated optimization of modulation parameter, transmission distance, energy harvesting rate and signal propagation loss for guaranteeing the perpetual nanonetworks and achieving the maximum network capacity.

In this paper, we analyze the tradeoff between energy harvesting and energy consumption for perpetual nanonetworks and then present the underlying constraints of the corresponding parameters. The main contributions of this paper are summarized as follows: First, the upper bound and lower bound of the transmitted pulse amplitude are presented from the aspects of limited harvested energy and SINR threshold, respectively. Moreover, the mathematical expression of SINR is also derived by considering the peculiarities of $\mathrm{THz}$ communication. Second, in order to guarantee the perpetual networks, the closed form of minimum spreading factor is explored through the comprehensive analysis of the energy harvesting-consumption tradeoff and TS-OOK scheme. Finally, the maximum achievable network capacity is investigated by jointly optimizing the parameters of spreading factor, transmission distance, pulse amplitude, pulse probability and node density while guarantee the perpetual nanonetworks. From the simulation results, short transmission distance and small spreading factor are recommended to achieve the maximum network capacity, while pulse probability is suggested from 0.31 to 0.46 under different network conditions.

The rest of this paper is organized as follows. In Section II, existing related works in pulse-based nanonetworks are reviewed, including the mechanism of TS-OOK and energy harvesting with piezoelectric nanogenerator. Section III presents the theoretical bounds of the transmitted pulse amplitude. In Section IV, multiple parameters are jointly optimized to achieve the maximum network capacity. In Section V, simulation results show the effects of multiple parameters on the performance of network capacity under different network conditions. Finally, the paper is concluded in Section VI.

\section{EXISTING RELATED WORKS IN Pulse-BASED NANONETWORKS}

Due to the limited capabilities of nanodevices, a pulsebased communication mechanism for nanonetworks is adopted by optimizing the spreading factor $\beta$, and the corresponding network capacity is given by considering the peculiarities of nanonetworks. In order to meet the energy requirement of pulse-based communication, an energy harvesting system by using piezoelectric nanogenerator is modeled as an ideal voltage source. Based on the existing circuit model of piezoelectric nanogenerator, the maximum harvested energy $E_{c a p-\max }$ and the energy harvesting rate $\lambda_{\text {harv }}$ in the nanocapacitors are presented, respectively.

\section{A. Pulse-Based Communication in Nanonetworks}

New information encoding and modulation mechanisms for nanonetworks are required to exploit the huge bandwidth provided by the $\mathrm{THz}$ Band. Compared with the existing complex modulations, Jornet and Akyildiz [12], [13] proposed a novel communication paradigm called TS-OOK for electromagnetic nanonetworks. This mechanism is based on the transmission of femtoseconds-long pulses by following an on-off keying modulation spread in time. In detail, a logical " 1 " is transmitted by using one-hundred-femtoseconds long pulse and a logical " 0 " is transmitted as silence, i.e., the nanodevices remain silent when a logical " 0 " is transmitted. In this paper, we consider the TS-OOK as the modulation mechanism to analyze the energy consumption for pulse-based nanonetworks due to its two advantages as shown in Fig. 1. First, it does not require the tight synchronization among the nanodevices all the time. Second, the channel can be shared without significant interference by multiple users when using TS-OOK.

Due to the constrained energy in nanodevices, short pulses cannot be emitted in a burst. In TS-OOK, the time $T_{S}$ between two consecutive symbols is much larger than the pulse duration $T_{p}$, i.e., the spreading factor $\beta=T_{S} / T_{p} \gg 1$. During the time between two symbols, nanodevices can either receive other incoming information flows or remain idle. For the purpose of relaxing the energy requirement in nanonetworks, the bigger value of $\beta$ is more preferable. However, a big $\beta$ will lead to low network capacity and long transmission delay because a lot of time slots are unutilized. Therefore, the spreading factor $\beta$ should be optimized by integrating the information of energy consumption rate, energy harvesting rate and available network capacity. Without considering the interference from other nanodevices, the single-user capacity $C_{s, u}$ in bits/s can be achieved in the $\mathrm{THz}$ Band is given by [12]

$$
C_{s, u}=\frac{B}{\beta} I R_{s, u}
$$

where $B$ refers to the bandwidth, $I R_{s, u}$ refers to the achievable information rate in bits/symbol for a single user system, it can be given as

$$
\begin{aligned}
I R_{S, u}=\max _{X}\left\{\left(-\sum_{m=0}^{1} p_{m} \log _{2} p_{m}-\int_{y} \sum_{m=0}^{1} \frac{p_{m} e^{-\frac{\left(y-a_{m}\right)^{2}}{2 N_{m}}}}{\sqrt{2 \pi N_{m}}}\right.\right. \\
\left.\left.\cdot \log _{2}\left(\sum_{n=0}^{1} \frac{p_{n}}{p_{m}} \sqrt{\frac{N_{m}}{N_{n}}} e^{-\frac{1}{2} \frac{\left(y-a_{n}\right)^{2}}{N_{n}}+\frac{1}{2} \frac{\left(y-a_{m}\right)^{2}}{N_{m}}}\right) d y\right)\right\}
\end{aligned}
$$




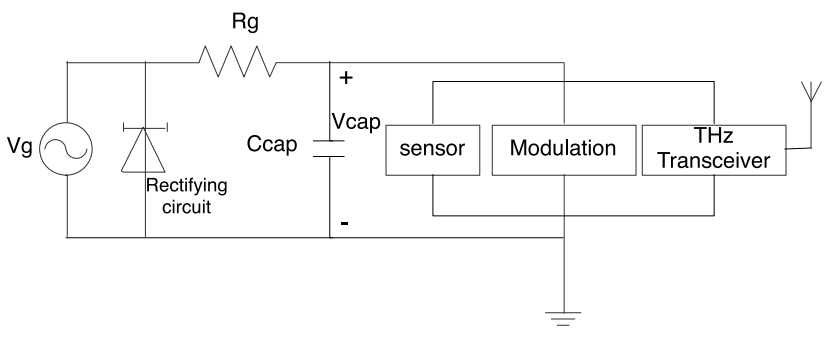

Fig. 2. Circuit model of piezoelectric nanogenerator.

where $p_{m}$ refers to the probability of transmitting the symbol $m, N_{m}$ refers to the total noise power associated with the transmitted symbol $m, a_{m}$ refers to the amplitude of the received symbol $m$. In order to guarantee the perpetual data transmission in nanonetworks, these parameters should be manipulated to maximize the network capacity while satisfying the energy requirements simultaneously. By increasing the spreading factor $\beta$, the maximum achievable information rate is reduced, but the energy requirement on the nanodevice is significantly relaxed. In this paper, the maximum achievable network capacity is investigated numerically by optimizing the parameters of spreading factor, the transmission distance and node density in Section V. The detailed computations of these parameters will be presented in the next sections.

\section{B. Energy Harvesting of Piezoelectric Nanogenerator}

In nanonetworks, the lifetime of each nanodevice is mainly dependent on the limited energy provided by the nanobatteries with a very small size. Hence, it is highly desirable that nanodevices are enabled to be self-powered without the use of nanobatteries. In the last five years, nanotechnology-enabled methods for converting mechanical energy into electrical energy by using the piezoelectric effect of Zinc Oxide nanogenerators have been explored [20]-[23]. Due to the low energy consumption of nanodevices during the transmission of information in the THz Band, especially over a short distance, the resultant energy harvested from the environment should be sufficient to power the nanodevices.

According to the simplified circuit model of piezoelectric nanogenerator as shown in Fig. 2, through several cycles of charging, the voltage of the nanocapacitor rises up, and the harvested energy is also stored in the nanocapacitor to power other modules in a nanodevice. Without loss of generality, it is assumed that all harvested energy can be stored in the array of nanocapacitors. The maximum energy $E_{c a p-\max }\left(n_{c y c}\right)$ stored in the nanocapacitors after a number of cycles $n_{c y c}$ can be computed as a function of the total capacitance $C_{c a p}$ and voltage $V_{\text {cap }}$ of the array of nanocapacitors:

$$
E_{c a p-\max }\left(n_{c y c}\right)=\frac{1}{2} C_{c a p}\left(V_{c a p}\left(n_{c y c}\right)\right)^{2} .
$$

For a particular nanodevice, its total capacitance $C_{c a p}$ and voltage $V_{\text {cap }}$ are always predefined, then the required number of charging cycles $n_{c y c}$ can be obtained when the energy consumption for transmission is determined, which will be comprehensively investigated in the next sections. Finally, the energy harvesting rate $\lambda_{\text {harv }}$ in Joule/second at which the capacitors are charged can be obtained as follows [15], [19]:

$$
\begin{aligned}
\lambda_{\text {harv }} & =\frac{1}{t_{c y c}} \cdot \frac{\partial E_{c a p}}{\partial n_{c y c}} \\
& =\frac{V_{g} \cdot \Delta Q}{t_{c y c}} \cdot\left(e^{\left(-\frac{\Delta Q \cdot n_{c y c}}{V_{g} C_{c a p}}\right)}-e^{\left(-2 \frac{\Delta Q \cdot n_{c y c}}{V_{g} C_{c a p}}\right)}\right)
\end{aligned}
$$

where $\Delta Q$ refers to the amount of electric charge obtained from one cycle, $\frac{\partial E_{c a p}}{\partial n_{c y c}}$ refers to the energy increase of the nanocapacitors in each cycle, and $t_{c y c}$ is the required time of one charging cycle, i.e., the time between two consecutive cycles. If the compress-release ${ }^{1}$ cycles are created by an artificially generated vibration source, the value of $t_{c y c}$ corresponds to the inverse of the frequency of the vibration source. In this paper, the minimum value of $n_{c y c}$ can be calculated to guarantee the required energy for transmission in the $\mathrm{THz}$ Band as shown in Section II-A.

\section{THEORETICAL BOUNDS OF THE Transmitted Pulse Amplitude}

In order to achieve the perpetual data transmission in the pulse-based nanonetworks, the tradeoff between energy harvesting and energy consumption needs to be investigated comprehensively. Based on the modulation of TS-OOK, the theoretical bounds of the amplitude of the transmitted pulse for perpetual data transmission are derived in this section.

\section{A. Upper Bound of the Transmitted Pulse Amplitude}

Due to the peculiarities of $\mathrm{THz}$ Band in nanonetworks, a big transmission power at the transmitter is desirable to overcome the severe path loss, while the total available energy is extremely constrained by each nanodevice. Hence, it is necessary to investigate the maximum transmission power that each nanodevice with energy harvesting can support.

According to the TS-OOK modulation, energy is only consumed for the transmission of pulses, not the silences. Moreover, the major spectral components of these pulses are constrained within the THz Band. Without loss of generality, these pulses can be modeled as Gaussian-shaped, which have been used in many applications such as Terahertz imaging and biological spectroscopy. The Gaussian pulse can be written as

$$
p(t)=\frac{a_{o}}{\sqrt{2 \pi} \sigma} e^{-(t-\mu)^{2} /\left(2 \sigma^{2}\right)}
$$

where $a_{o}$ refers to the amplitude of the transmitted pulse, which can be used to adjust the pulse transmission power. $\sigma$ is the standard deviation of the Gaussian pulse in seconds, and $\mu$ is the location in time for the center of the pulse in seconds. The p.s.d. of the time derivative of a Gaussian pulse is also Gaussian-shaped, but the frequency position of its main component increases with the derivative order $n$. Thus, the p.s.d. of the transmitted pulse $S_{p}^{(n)}(f)$ can be calculated as follows [17]

$$
S_{p}^{(n)}(f)=(2 \pi f j)^{2 n} a_{0}^{2} e^{-(2 \pi f \sigma)^{2}}
$$

\footnotetext{
${ }^{1}$ In detail, an electric flow (current) is generated between the ends of nanowires as they are compressed, and this current is utilized to charge the nanocapacitors or directly power other modules in a nanodevice. The rectifying circuit is used to adjust the current to charge the nanocapacitors because of the opposite direction of the generated current as the nanowires are released.
} 
where $f$ refers to the transmission frequency (in Hertz). In this paper, the transmitted pulse is defined as the first timederivative of a one-hundred-femtoseconds-long Gaussian pulse because the antenna in nanodevice cannot radiate the pulse with a strong DC component, i.e., $n=1$. Based on the above derived p.s.d. of Gaussian pulse, the transmission power of transmitting one pulse $P_{t x}^{\text {pulse }}$ can be written as

$$
P_{p u l s e}^{t x}=\int_{B} S_{p}^{(1)}(f) d f=a_{0}^{2} \int_{B}(2 \pi f)^{2} e^{-(2 \pi f \sigma)^{2}} d f
$$

where $B$ refers to the bandwidth of the transmitted signal. Therefore, the calculation of maximum available transmission power can be addressed by determining the upper bound of the amplitude of the transmitted pulse.

Since the limitation of maximum energy harvested by nanogenerator, for transmitting one packet with TS-OOK mechanism, the minimum required energy to guarantee one pulse transmission can be obtained at each nanodevice, i.e., $\left(P_{\text {pulse }}^{t x} T_{p}+P_{\text {circuits }} T_{s}\right) . P_{\text {circuits }}$ is made up of two components according to its definition, i.e., power consumption of the transmitter circuit $P_{t x \text {, circuit }}$ and the receiver circuit $P_{r x, \text { circuit }} . P_{\text {circuits }}$ is used to perform the modulation and any other processing, and its value is independent on the transmission distance between the nanodevices or the amount of energy radiated into the channel by the antenna. It means, for each self-powered nanodevice, the harvested energy after $n_{c y c}$ cycles should be enough to satisfy the energy requirement of transmitting one packet over one hop, i.e., at least the value of $E_{c a p-\max }\left(n_{c y c}\right)$, as shown in (3), must be greater than the value of the minimum required energy for one packet transmission. In detail, this relationship corresponds to

$$
\begin{aligned}
& \frac{1}{2} C_{c a p} V_{g}^{2}\left(1-e^{\left(-\frac{n_{c y c} \cdot \Delta Q}{V_{g} \cdot C_{c a p}}\right)}\right)^{2} \\
& \quad \geq N_{p}\left(P_{\text {pulse }}^{t x} T_{p} p(1)+P_{\text {circuits }} T_{s}\right)
\end{aligned}
$$

where $p(1)$ refers to the probability of transmitting pulses, $N_{p}$ refers to the length of one packet in bits.

Therefore, the minimum number of cycles $n_{c y c}^{\mathrm{min}}$ needed to charge the nanocapacitors up to the required energy is obtained from (8)

$$
\begin{aligned}
n_{c y c}^{\min } \geq & \frac{V_{g} C_{c a p}}{\Delta Q} \\
& \left(1-\ln \left(1-\left(\frac{2 N_{p}\left(P_{\text {pulse }}^{t x} T_{p} p(1)+P_{\text {circuits }} T_{s}\right)}{C_{\text {cap }} V_{g}^{2}}\right)\right) .\right.
\end{aligned}
$$

For various power sources, the processes of charging nanocapacitors have a little difference. For example, if the charging cycles of nanowires are generated by an ambient vibration, such as the vents of the air conditioning system of an office or body movement, the arrivals of these cycles always follow a Poisson Distribution [24], [25]; if the charging cycles of nanowires are generated by an ultrasonic wave with fixed frequency, the arrivals of these cycles correspond to the inverse of that frequency [26]. Hence, the required time to charge the nanocapacitors up to the required energy can be obtained by integrating the charging frequency and the minimum charging cycles $n_{c y c}^{\mathrm{min}}$.

However, in order to guarantee the effective value of $n_{c y c}^{\min }$, there is an underlying constraint in (9), i.e., 1 $\left(\frac{2 N_{p}\left(P_{\text {pulse }}^{t x} T_{p} p(1)+P_{\text {circuits }} T_{s}\right)}{C_{\text {cap }} V_{g}^{2}}\right)^{\frac{1}{2}} \geq 0$. Through the simplification, it can be transferred to an underlying constraint of $P_{\text {pulse }}^{t x}$ as follows

$$
N_{p} P_{\text {pulse }}^{t x} T_{p} p(1) \leq \frac{1}{2} C_{\text {cap }} V_{g}^{2}-N_{p} P_{\text {circuits }} T_{s}<\frac{1}{2} C_{\text {cap }} V_{g}{ }^{2} .
$$

The physical meaning of (10) is that, for each self-powered nanodevice, the value of energy consumption for transmitting one packet should not be greater than half of the total harvested energy by the nanowires after $n_{c y c}^{\min }$ cycles. Based on the p.s.d. of the transmitted pulse, the upper bound of the transmitted pulse amplitude $a_{\max }$ can be obtained as follows

$$
P_{\text {pulse } \max }^{t x}=a_{\max }^{2}<\frac{C_{c a p} V_{g}^{2}}{2 N_{p} T_{p} p(1)} .
$$

For the time being, since no specific technology has been considered to implement the transceiver of nanodevice, the energy consumption model of transceiver is not available; thus, we focus on the energy consumed to overcome the channel attenuation, i.e., spreading loss and molecular absorption loss in this paper, and assume the energy consumption of transceiver circuit is fixed [15], [19].

\section{B. Lower Bound of the Transmitted Pulse Amplitude}

From the view of the receiver, the transmitted signal can be received successfully only when the received signal power is beyond the predefined constant Signal-to-Interference-Noise Ratio (SINR). In practice, when silences are transmitted, the energy consumption at the transmitter is not required for transmitting signal at the antenna, only required for other processing modules. Therefore, for one transmitted pulse in the TS-OOK, the transmission power $P_{\text {pulse }}^{t x}$ spent at the transmitter can also be computed as the power required in the transmission to overcome the spreading loss and molecular absorption loss, and finally guarantee the constant SINR at the receiver.

Each transmitted signal suffers the propagation attenuation during the transmission, its path loss consists of spreading loss and molecular absorption loss. Thus, the relationship between the p.s.d. of the transmitted pulse and the corresponding received power $P_{p u l s e}^{r x}(d)$ can be given as follows:

$$
P_{p u l s e}^{r x}(d)=\int_{B} S_{p}^{(1)}(f)\left|H_{c}(f, d)\right|^{2}\left|H_{r}(f)\right|^{2} d f,
$$

where $H_{r}(f)$ refers to the receiver frequency response, which is considered as an ideal low-pass filter with bandwidth $B, d$ is the transmission distance (in Meter). $H_{c}(f, d)$ refers to the $\mathrm{THz}$ Band channel frequency response during the transmission over the distance $d$, which is given by [11] and [17]

$$
\begin{aligned}
H_{c}(f, d) & =H_{s p r}(f, d) H_{a b s}(f, d) \\
& =\left(\frac{c}{4 \pi d f}\right) \exp \left(-\frac{\alpha(f) d}{2}\right)
\end{aligned}
$$


where $H_{a b s}(f, d)$ and $H_{s p r}(f, d)$ refer to the molecular absorption loss and spreading loss, respectively. The absorption coefficient $\alpha(f)$ is a function of transmission frequency of the electromagnetic wave, and it is also dependent on the composition of the medium, i.e., the particular mixture of molecules found along the path,

$$
\alpha(f)=-\sum_{i, g} \frac{P}{P_{0}} \frac{T_{0}}{T} \cdot Q_{i, g} \cdot \sigma_{i, g}(f) \cdot d
$$

For a standard medium, oxygen $\left(\mathrm{O}_{2}, 20.9 \%\right)$ and water vapor $\left(\mathrm{H}_{2} \mathrm{O}, 1 \%\right)$ have resonance frequencies in the THz Band, while the resonance frequency of nitrogen $\left(N_{2}, 78.1 \%\right)$ is beyond the THz Band. Each gas has different resonating isotopologues at several frequencies within the $\mathrm{THz}$ Band. $P_{o}$ and $T_{o}$ are the standard pressure and temperature values, $P$ and $T$ are the pressure and temperature values and $\sigma_{i, g}(f)$ is the absorption cross section for the isotopologue $i$ of gas $g$ in $\mathrm{m}^{2} /$ molecule. $Q_{i, g}$ is the total number of molecules per volume of the isotopologue $i$ of gas $g$ in a unit of molecule $/ \mathrm{m}^{3}$. According to the radiative transfer theory and the information provided by the widely adopted HITRAN database (HIgh resolution TRANsmission molecular absorption database), all the above variables can be directly or indirectly obtained.

According to the definition of SINR, it can be calculated in the $\mathrm{THz}$ Band as follows

$$
\operatorname{SINR}=\frac{P_{p u l s e}^{r x}(d)}{N_{p}(d)+N_{I_{p}}}=\frac{\int_{B} S_{p}^{(1)}(f)\left|H_{c}(f, d)\right|^{2}\left|H_{r}(f)\right|^{2} d f}{\int_{B} S_{N_{p}}(f, d)\left|H_{r}(f)\right|^{2} d f+N_{I_{p}}}
$$

where $N_{p}(d)$ and $N_{I_{p}}$ refer to the noise and interference power associated with the transmitted pulse, respectively. The total noise in the $\mathrm{THz}$ Band is contributed by the background atmospheric noise and the self-induced noise, which can be obtained as follows [29]

$$
\begin{aligned}
N_{p}(d) & =\int_{B} S_{N_{P}}(f, d)\left|H_{r}(f)\right|^{2} d f \\
& =\int_{B}\left(S_{N^{B}}(f)+S_{N_{p}^{I}}(f, d)\right)\left|H_{r}(f)\right|^{2} d f,
\end{aligned}
$$

where $S_{N_{P}}(f, d)$ refers to the p.s.d. of the total noise given the transmission of pulse. $S_{N^{B}}(f)$ and $S_{N_{p}^{I}}(f, d)$ refer to the p.s.d. of the background atmospheric noise and the self-induced noise by transmitting pulses, respectively. In detail, they are defined correspondingly on the basis of channel response as

$$
\begin{aligned}
S_{N^{B}}(f)= & \lim _{d \rightarrow \infty} k_{B} T_{0}\left(1-\left|H_{a b s}(f, d)\right|^{2}\right)\left|H_{a n t}^{R}(f)\right|^{2} \\
= & \lim _{d \rightarrow \infty} k_{B} T_{0}(1-\exp (-\alpha(f) d))\left(\frac{c}{\sqrt{4 \pi} f_{0}}\right)^{2}, \\
S_{N_{p}^{I}}(f, d)= & S_{p}^{(1)}(f)\left|H_{a n t}^{T}(f)\right|^{2}\left(1-\left|H_{a b s}(f, d)\right|^{2}\right) \\
& \cdot\left|H_{s p r}(f, d)\right|^{2}\left|H_{a n t}^{R}(f)\right|^{2} \\
= & S_{p}^{(1)}(f)(1-\exp (-\alpha(f) d))\left(\frac{c}{4 \pi d f_{0}}\right)^{2}
\end{aligned}
$$

where $k_{B}$ refers to the Boltzmann constant, $T_{0}$ refers to the room temperature, $H_{\text {ant }}^{T}(f)$ and $H_{\text {ant }}^{R}(f)$ refer to the antenna frequency response at the transmitter and receiver, respectively [11], [12]. $f_{0}$ is the center frequency of the transmitted pulse.

On the other hand, the main constraints in nanonetworks, i.e., severe path loss of $\mathrm{THz}$ Band and limited energy of each nanodevice, result in the extremely short transmission distance, and which also leads to the high density of nanodevices to guarantee the communications. Therefore, multi-user interference should be taken into consideration to evaluate the performance of nanonetworks. According to the TS-OOK mechanism, each nanonode can start transmitting symbols at any time in an uncoordinated manner, it may cause the collisions between the transmitted symbols. For a typical receiver, the interference occurs when multiple symbols from different nanonodes arrive at the same time, which contributes to the overlap of the amplitude and shape of the transmitted symbols. In particular, collisions between the transmitted silences are not harmful. Collisions between the transmitted pulses and silences are only harmful to the transmitted silences.

Without loss of generality, the transmitted symbols from different nanonodes are assumed to be independent and follow the same distribution of source probability. Moreover, due to the high density of nanonetowrks, transmissions from different nanonodes can be regarded as uniformly distributed in time by selecting a random waiting time before transmitting the symbols at nanonodes. For a typical receiver, the amplitude of its total interference is dependent on all interfering nodes with corresponding propagation conditions and distances. Therefore, under the high traffic condition, the interference can be modeled as a Gaussian random process, i.e., $\mathcal{N}_{I_{p}}\left(\mu_{I_{p}}=E\left[I_{p}\right] ; \sigma_{I_{p}}^{2}=N_{I_{p}}\right)$. For the transmitted symbols from a typical nanonode, the average interference $E\left[I_{p}\right]$ at the corresponding receiver is given by [12]:

$$
E\left[I_{p}\right]=\sum_{i \in \Phi_{j}} \frac{T_{p}}{T_{s}} a^{i, j} p_{j}(1)=\sum_{i \in \Phi_{j}} a^{i, j} p_{j}(1) / \beta
$$

where $i$ refers to the interfering node, $\Phi_{j}$ refers to the set of interfering nodes of the receiver $j, a^{i, j}$ refers to the average pulse amplitude at the receiver $j$, transmitted from the interfering node $i . p_{j}(1)$ indicates the source probability of transmitting pulses, and assume all nodes have the same source probability, i.e., $p_{j}(1)=p(1)$. The variance of the interference $N_{I_{p}}$ can be obtained as

$$
\begin{aligned}
N_{I_{p}}=\sum_{i \in \Phi_{j}}\left(\frac{\left(a^{i, j}\right)^{2}+N^{i, j}}{\beta}\right) & p(1)-\left(\sum_{i \in \Phi_{j}} \frac{a^{i, j}}{\beta} p(1)\right)^{2} \\
& +2 \sum_{i, k \in \Phi_{j}}\left(\frac{p(1)}{\beta}\right)^{2} a^{i, j} a^{k, j}
\end{aligned}
$$

where $N^{i, j}$ refers to the noise power generated by the transmission between node $i$ and node $j$. According to the noise model in (16), $N^{i, j}$ equals to $N_{p}\left(d_{i, j}\right)$, in which $d_{i, j}$ refers to the transmission distance between node $i$ and node $j$. In the frequency domain, the power of a received pulse is proportional to its amplitude squared, i.e., $a^{i, j}=\sqrt{P_{p u l s e}^{r x}\left(d_{i j}\right)}$, where the proportionality constant is assumed to be one. Thus, the 
SINR can be rewritten as follows in (21), as shown at the bottom of this page.

In order to guarantee the successful reception of the transmitted symbol at the side of receiver, its SINR value should be greater than the minimum threshold determined by the structure of nanodevice. Due to the high density of nanonetowrks, suppose that all interfering nodes approximately have the identical distance from the receiver, i.e., $d_{i j}=d$. Thus, $N^{i, j}=$ $N_{p}(d)$ refers to the average noise power and $a^{i, j}=\sqrt{P_{p u l s e}^{r x}(d)}$ refers to the average amplitude when transmitting pulses over the distance $d$. Therefore, the interference power $N_{I_{p}}$ with $U$ interfering nodes can be simplified as

$$
N_{I_{p}}=\frac{U p(1)}{\beta} P_{\text {pulse }}^{r x}\left(1+\frac{N_{p}(d)}{P_{p u l s e}^{r x}}+(2-U) \frac{p(1)}{\beta}\right)
$$

By combining the (15), (16) and (22), the SINR should be satisfied with the minimum SINR threshold $T$ as SINR= $\frac{P_{p u l s e}^{r x}}{N_{p}(d)+N_{I_{p}}} \geq T$. Thus, the received pulse power can be computed as

$$
\begin{array}{r}
P_{p u l s e}^{r x} \geq N_{p}(d) T+\frac{U p(1)}{\beta} T\left(1+(2-U) \frac{p(1)}{\beta}\right) P_{p u l s e}^{r x} \\
+\frac{U p(1)}{\beta} N_{p}(d) T .
\end{array}
$$

Through the simplification of the above equation, the minimum received pulse power can be obtained as

$$
P_{p u l s e}^{r x}(d) \geq \frac{N_{p}(d) T+\frac{U p(1)}{\beta} N_{p}(d) T}{1+\frac{U p(1)}{\beta^{2}} T(\beta+(2-U) p(1))}
$$

With (7) and (12), and based on the p.s.d. of the transmitted pulse, the lower bound of the transmitted pulse $a_{\text {min }}$ can be obtained.

\section{PARAMETER OPTIMIZATION FOR MAXIMIZING THE NETWORK CAPACITY}

Aimed at achieving the perpetual data transmission for pulse-based nanonetworks, the tradeoff between energy consumption rate and energy harvesting rate of each nanonode should be investigated comprehensively. Firstly, the data rate over each link should not be greater than the capacity of each node by considering the interference in the multi-user scenario. Secondly, the energy harvesting rate should be greater than the energy consumption rate by using the TS-OOK modulation. Based on the theoretical bounds of the amplitude of the transmitted pulse derived in the above section, the parameters, such as spreading factor $\beta$, transmission distance, node density and pulse probability, are required be comprehensively optimized to maximize the available network capacity.

\section{A. Minimum Spreading Factor for Perpetual Nanonetworks}

To better understand the correlation between the energy harvesting and consumption in nanonetworks, we firstly introduce the average energy consumption rate $\lambda_{\text {con }}$ for data transmission in $\mathrm{J} / \mathrm{s}$. According to the TS-OOK modulation, transmission energy is only consumed while transmitting pulses. In practice, pulses (signal "1") and silences (signal "0") are not transmitted in a burst, but spread in time. Furthermore, according to the analysis of pulse-based communication in Section II-A, a big value for spreading factor $\beta$ is recommended, which can guarantee the channel sharing while reducing the collisions among nanodevices. Thus, the energy consumption rate of each node can be obtained as follows

$$
\lambda_{\text {con }}=E_{\text {pulse }}^{t x} p(1) \frac{\lambda_{\text {bit }}}{\beta}=P_{\text {pulse }}^{t x} T_{p} p(1) \frac{\lambda_{b i t}}{\beta}
$$

where $E_{\text {pulse }}^{t x}$ refers to the required energy to transmit one pulse, $\lambda_{\text {bit }}$ refers to the available data rate of each node. The maximal energy consumption rate can be achieved when the data rate of each node approaches to its maximum transmission bit-rate $C_{n e t} / U$ in the multi-user systems. By considering the interference of multiple nanonodes in TS-OOK scheme [12], the network capacity $C_{n e t}$ in bits/second as the aggregated throughput of all nanonodes that can be achieved in the $\mathrm{THz}$ Band as shown in (26), as shown at the bottom of the next page.

Therefore, in order to guarantee the perpetual data transmission, the maximum energy consumption rate $\lambda_{\text {con-max }}$ (when $\lambda_{\text {bit }}=C_{n e t} / U$ ) should not exceed the energy harvesting rate $\lambda_{\text {harv }}$ given by (4). Thus, the relationship between energy harvesting rate and consumption rate at a nanodevice can be written by

$$
\lambda_{\text {harv }} \geq\left.\lambda_{\text {con }}\right|_{\lambda_{\text {bit }}=C_{\text {net }} / U}=E_{\text {pulse }}^{t x} p(1) \frac{C_{\text {net }}}{\beta U} .
$$

Therefore, through the simplification of the above equation, the spreading factor in TS-OOK for perpetual nanonetworks should be satisfied by

$$
\begin{aligned}
& \beta \geq \frac{E_{\text {pulse }}^{t x} p(1) C_{n e t}}{U \lambda_{\text {harv }}} \\
& =\frac{P_{p u l s e}^{t x} T_{p} p(1) C_{n e t} t_{c y c}}{U V_{g} \Delta Q\left(e^{\left(-\frac{\Delta Q n_{c y c}}{V_{g} C_{c a p}}\right)}-e^{\left(-2 \frac{\Delta Q n_{c y c}}{V_{g} C_{c a p}}\right)}\right)}
\end{aligned}
$$

$$
\begin{aligned}
\operatorname{SINR}= & \frac{\int_{B} S_{p}^{(1)}(f)\left|H_{c}(f, d)\right|^{2}\left|H_{r}(f)\right|^{2} d f}{\int_{B}\left(S_{N_{p}}(f)\right)\left|H_{r}(f)\right|^{2} d f+N_{I_{p}}} \\
= & \frac{\int_{B} S_{p}^{(1)}(f)\left|H_{c}(f, d)\right|^{2}\left|H_{r}(f)\right|^{2} d f}{\int_{B}\left(S_{N^{B}}(f)+S_{N_{p}^{I}}(f, d)\right)\left|H_{r}(f)\right|^{2} d f+\sum_{i \in \Phi_{j}}\left(\frac{\left(a^{i, j}\right)^{2}+N^{i, j}}{\beta}\right) p(1)-\left(\sum_{i \in \Phi_{j}} \frac{a^{i, j}}{\beta} p(1)\right)^{2}+2 \sum_{i, k \in \Phi_{j}}\left(\frac{p(1)}{\beta}\right)^{2} a^{i, j} a^{k, j}}
\end{aligned}
$$


where the transmission power $P_{\text {pulse }}^{t x}$ in TS-OOK is constrained by the theoretical bounds of the pulse amplitude in Section III. On the one hand, the network capacity $C_{n e t}$ and the multi-user interference are both dependent on the spreading factor $\beta$. Therefore, it is not easy to obtain the analytical expression of spreading factor in the multi-user systems. On the other hand, for one single user systems, there is no multi-user interference, i.e., $C_{s, u}=\frac{B}{\beta} I R_{s, u} \geq C_{\text {net }} / U$, then the minimum spreading factor in TS-OOK can be written approximately as

$$
\begin{aligned}
\beta_{\min } & \geq\left(\frac{E_{\text {pulse }}^{t x} p(1) I R_{s, u} B}{\lambda_{\text {harv }}}\right)^{\frac{1}{2}} \\
& =\left(\frac{P_{p u l s e}^{\text {tx }} T_{p} p(1) I R_{s, u} B t_{c y c}}{V_{g} \Delta Q\left(e^{\left(-\frac{\Delta Q n_{c y c}}{V_{g} C_{c a p}}\right)}-e^{\left(-2 \frac{\Delta Q n_{c y c}}{V_{g} C_{c a p}}\right)}\right)}\right)^{\frac{1}{2}} .
\end{aligned}
$$

\section{B. Maximization of Network Capacity}

Due to the strict constraints of limited energy and high transmission frequency, the network capacity presented in (26) is dependent on the parameters of spreading factor, multi-user interference, transmission distance, pulse amplitude, source probability, SINR threshold and node density. To the state of the art, little research has been conducted to investigate the capacity of nanonetworks in $\mathrm{THz}$ Band, while taking all these parameters into consideration for perpetual nanonetworks [30]. Therefore, it is necessary to optimize the above system parameters to maximize the network capacity while guaranteeing the perpetual communication in nanonetworks with energy harvesting.

For the different constraints and complex relationships of multiple parameters, the maximization problem of network capacity can be presented as follows

$$
\begin{array}{ll}
\max & C_{\text {net }}(\beta, U, a, d, T, p(1)) \\
\text { s.t. } & P_{p u l s e, \max }^{t x}=a_{\max }^{2}<\frac{C_{c a p} V_{g}^{2}}{2 N_{p} T_{p} p(1)} \\
& P_{p u l s e}^{r x}(d) \geq \frac{N_{p}(d) T+\frac{U p(1)}{\beta} N_{p}(d) T}{1+\frac{U p(1)}{\beta^{2}} T(\beta+(2-U) p(1))}
\end{array}
$$

$$
\beta_{\min } \geq\left(\frac{P_{p u l s e}^{t x} T_{p} p(1) I R_{s, u} B t_{c y c}}{V_{g} \Delta Q\left(e^{\left(-\frac{\Delta Q n_{c y c}}{V_{g} C_{c a p}}\right)}-e^{\left(-2 \frac{\Delta n_{c y c}}{V_{g} C_{c a p}}\right)}\right)}\right)^{\frac{1}{2}}
$$

It is observed that the maximum amplitude of the transmitted pulse $a_{\max }$ depends on the structure of the energy harvesting system, while the minimum amplitude relies on the propagation channel, such as noise, spreading factor, node density, source probability and transmission distance. Moreover, the minimum spreading factor is dependent on the amplitude of the transmitted pulse, source probability, energy harvesting system and network capacity. In the next section, the effects of all these parameters on network capacity will be comprehensively evaluated, and achieve the maximum network capacity under the specific conditions.

\section{Simulations AND ANALYsis}

In this section, the joint parameter optimization for maximizing the capacity of pulse-based nanonetworks is comprehensively investigated through extensive simulations. Without loss of generality, the transmission medium is assumed to be composed of nitrogen $(78.1 \%)$, oxygen $(20.9 \%)$ and water vapor $(1 \%)$ in the simulations. Due to the severe path loss of the $\mathrm{THz}$ Band, transmission distance is varied from $0.1 \mathrm{~mm}$ to $1 \mathrm{~m}$. The probability of transmitting pulses $p(1)$ equals to 0.5 . The transmitted pulse duration $T_{p}$ is 100 femtoseconds. The SINR threshold $T$ is fixed as $10 \mathrm{~dB}$.

\section{A. Theoretical Bounds of Transmitted Pulse Amplitude}

According to the circuit model of piezoelectric nanogenerator as shown in Fig. 2, a current rectification is adopted to fully use the electrical energy harvested in one full cycle of mechanical deformation from both pressing and releasing [20]. To obtain realistic values of the maximum energy $E_{c a p-\max }$ and the minimum number of cycles $n_{c y c}^{\min }$, feasible values for the parameters $\Delta Q, V_{g}$ and $C_{c a p}$ need to be determined. In this paper, the harvested energy is stored consecutively by connecting eight $22 \mu \mathrm{F}$ nanocapacitors in parallel at a voltage $V_{g}=0.42 \mathrm{~V}$, i.e., the total capacitance $C_{c a p}=176 \mu \mathrm{F}$, and the total electric charge per cycle is $\Delta Q=3.63 \mathrm{nC}$ [15], [19]. In the worst case, based on the above parameter values, the minimum cycles $n_{c y c}^{\min }$ needed to charge the nanocapacitors up to guarantee the required energy of transmitting one packet is

$$
\begin{aligned}
C_{n e t}=\max _{X}\left\{\frac { U B } { \beta } \left(-\sum_{m=0}^{1} p_{m} \log _{2} p_{m}-\int \sum_{m=0}^{1} \frac{e^{-\frac{\left(y-E[I]-a_{m}\right)^{2}}{2\left(N_{m}+N_{I}\right)}}}{\sqrt{2 \pi\left(N_{m}+N_{I}\right)}} p_{m}\right.\right. \\
\left.\left.\cdot \log _{2}\left(\sum_{n=0}^{1} \frac{p_{n}}{p_{m}} \sqrt{\frac{N_{m}+N_{I}}{N_{n}+N_{I}}} e^{-\frac{1}{2} \frac{\left(y-E[I]-a_{n}\right)^{2}}{N_{n}+N_{I}}+\frac{1}{2} \frac{\left(y-E[I]-a_{m}\right)^{2}}{N_{m}+N_{I}}}\right) d y\right)\right\}
\end{aligned}
$$




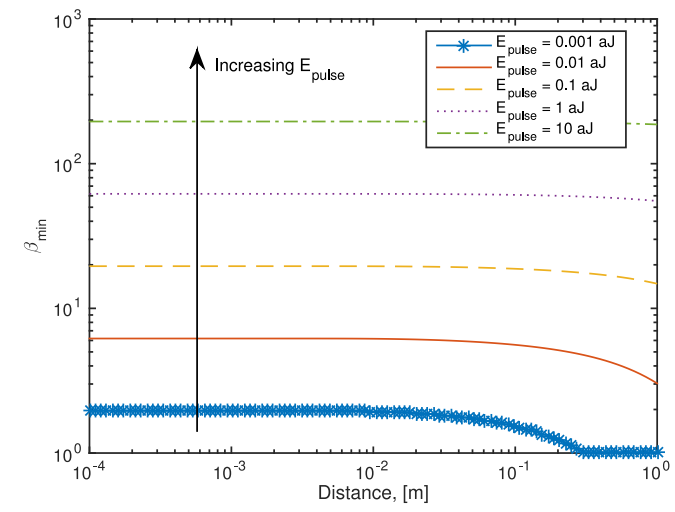

Fig. 3. Minimum spreading factor for perpetual nanonetworks with different transmitted pulse energy, $\beta_{\min }$.

in the order of $10^{4}$ cycles. In detail, when the pulse energy is set as 1 aJ, the minimum cycles $n_{c y c}^{\min }$ needed to charge the nanocapacitor is close to $2.0364 \times 10^{4}$, which can also be proved by the experimental results in [15], [19], and [20].

Based on the measured values of $V_{g}=0.42 \mathrm{~V}$ and $C_{c a p}=176 \mu \mathrm{F}$, the underlying constraint in (10), i.e., the required energy of transmitting one pulse should be smaller than $3.1 \times 10^{-8} \mathrm{~J}$, and the corresponding upper bound of pulse amplitude closes to $557 \mathrm{~V}$ when $N_{p}=1000$ bits. On the other hand, the lower bound of the transmitted pulse amplitude is constrained by the (24), the minimum reception power as well as the lower bound of pulse amplitude decrease with the addition of transmission distance as a result of the reduction of interference and noise power. The lower bound of the transmitted pulse amplitude is around $7.17 \times 10^{-8} \mathrm{~V}$ as the transmission distance is $0.1 \mathrm{~mm}$.

\section{B. Minimum Spreading Factor for Perpetual Nanonetworks}

Due to the severe path loss of $\mathrm{THz}$ Band, the achievable information rate decreases with the transmission distance as a result of low received signal power at the receiver. Therefore, by utilizing the same energy harvesting parameters, the minimum spreading factor $\beta_{\min }$ in (29) is reduced with the increase of transmission distance when the transmitted pulse energy is identical as shown in Fig. 3. In detail, a small value of $\beta$ indicates a high transmission probability (the time between two consecutive symbols becomes short) based on the TS-OOK modulation. Therefore, more pulses are required to be transmitted under the condition of longer transmission distance. On the other hand, when the transmission distance is identical, the minimum spreading factor increases dramatically with the addition of transmitted pulse energy. For example, as the pulse energy is changed from 1 aJ to 10 aJ, the corresponding minimum spreading factor needs to be enlarged from 62 to 196, which means the nanodevice needs more time for harvesting energy to transmit pulse with a relative big energy. Moreover, the information rate achieves a high value (i.e., approaches to $1 \mathrm{bit} / \mathrm{symbol}$ ) when transmitting pulse with a big energy, and it contributes to a similar value of $\beta_{\min }$, which is not affected by the short transmission distance.

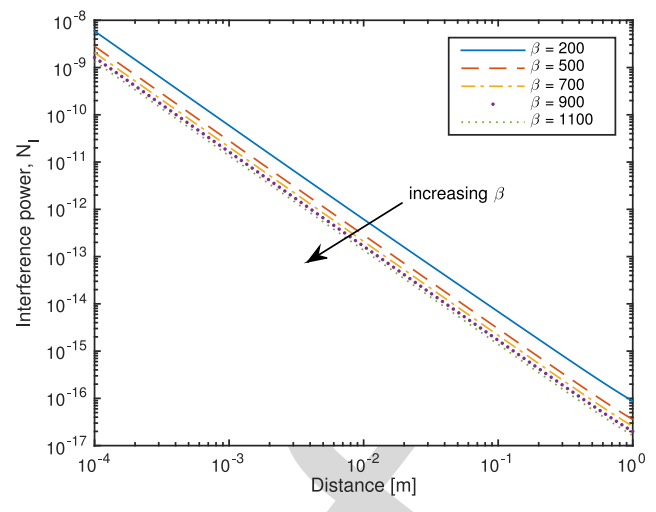

Fig. 4. Interference power $N_{I}$.

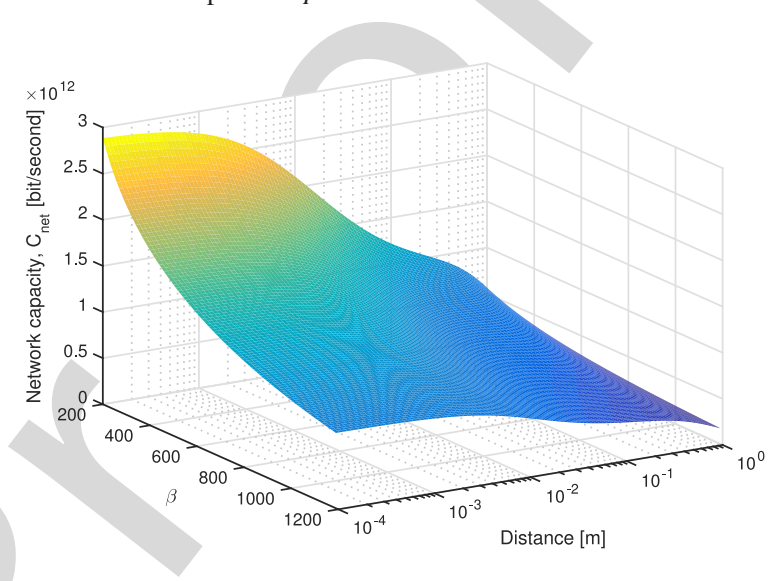

Fig. 5. Network capacity $C_{n e t}($ when $p(1)=0.5$ and $U=100)$.

In the multi-user nanonetworks, the interference with different value of $\beta$ should be evaluated while a big spreading factor is recommended to relieve the energy requirement in nanonetowrks. According to the above analysis, the minimum spreading factor is dependent on the transmitted pulse energy and transmission distance. Furthermore, we analyze the relationship between the interference distribution and the value of spreading factor $\beta$ (greater than $\beta_{\min }$ ) over different transmission distances. When each transmitted pulse energy is set as $1 \mathrm{aJ}$, the average interference in (22) is reduced with the increase of spreading factor, i.e., more time slots are unutilized to transmit symbols in the pulse-based nanonetworks. For a smaller spreading factor $\beta$, more pulses are transmitted from nanodevices in the same time period, which results in the higher interference as shown in Fig. 4. Moreover, the interference decreases with the transmission distance as a result of higher path loss with longer distance.

\section{Network Capacity}

In order to guarantee the perpetual data transmission, the spreading factor should be satisfied by the constraint in (29). Moreover, the transmission distance, pulse probability and the number of nanonodes are also required to be comprehensively manipulated to maximize the achievable network capacity. Given the fixed pulse probability $(p(1)=0.5)$ and node density $(U=100)$, Fig. 5 shows the effects of spreading factor and transmission distance on network capacity, it is 


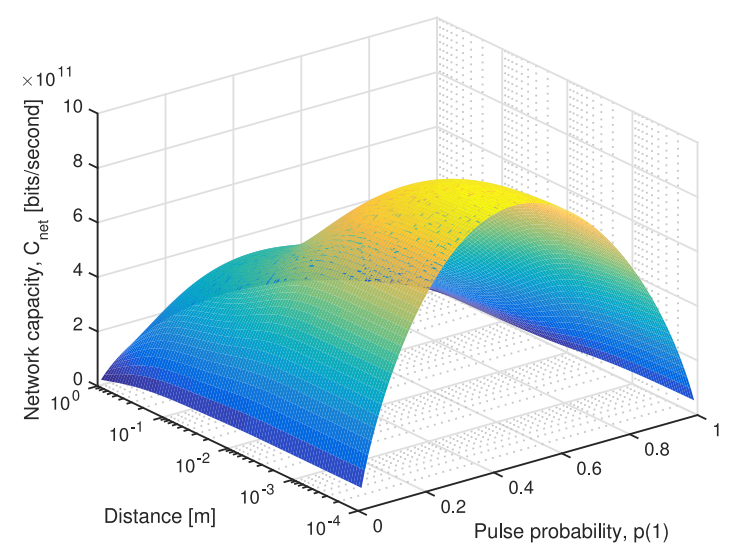

Fig. 6. Network capacity $C_{\text {net }}$ (when $\beta=1000$ and $U=100$ ).

observed that short transmission distance and small spreading factor (greater than $\beta_{\min }$ ) are recommended to achieve high network capacity and satisfy the energy requirement. Particularly, the shorter transmission distance the lower path loss, which contributes to the bigger information rate. Small value of spreading factor results in more transmitted symbols as well as introduces more interference, but the average interference power is still very low in the case of non-dense nanonetworks $U=100$ as shown in Fig. 4. Finally, the performance of network capacity is degraded with the increase of transmission distance and spreading factor.

From the aspects of energy consumption and interference power, pulse probability has a main role on the performance of network capacity. Fig. 6 shows the relationship between the pulse probability and network capacity over different transmission distances. It is observed that when the number of nanonodes is fixed as 100 , the maximum network capacity is achieved at $C_{n e t}=9.59 \times 10^{11} \mathrm{bits} / \mathrm{second}$ as the pulse probability approximately equals to 0.46 and transmission distance is $0.1 \mathrm{~mm}$, while the maximum network capacity is changed to $C_{\text {net }}=7.48 \times 10^{11} \mathrm{bits} / \mathrm{second}$ as the pulse probability closes to 0.41 and transmission distance is $10 \mathrm{~mm}$. It means that under different transmission distances, there are the corresponding best pulse probabilities $p(1)_{\text {best }}$ to maximize the network capacity. When the transmission distance is fixed, with the increase of $p(1)$, the achievable network capacity is increased as the $p(1)<p(1)_{\text {best }}$ in the light of additional information rate, and is reduced as the $p(1)>p(1)_{\text {best }}$ due to the incremental high probability of collision between the transmitted pulses. Finally, it is concluded that the value of pulse probability is recommended to be reduced with the increase of transmission distance to improve the network capacity, and the best value of pulse probability is dependent on the spreading factor and transmission distance. When the transmission distance increases from $0.1 \mathrm{~mm}$ to $1 \mathrm{~m}$, the corresponding most appropriate pulse probability is changed from 0.35 to 0.46 to guarantee the maximum network capacity.

Furthermore, the effects of pulse probability and spreading factor on the network capacity are illustrated in Fig. 7. When the transmission distance and node density is fixed, the pulse probability has a main role in achieving the maximum

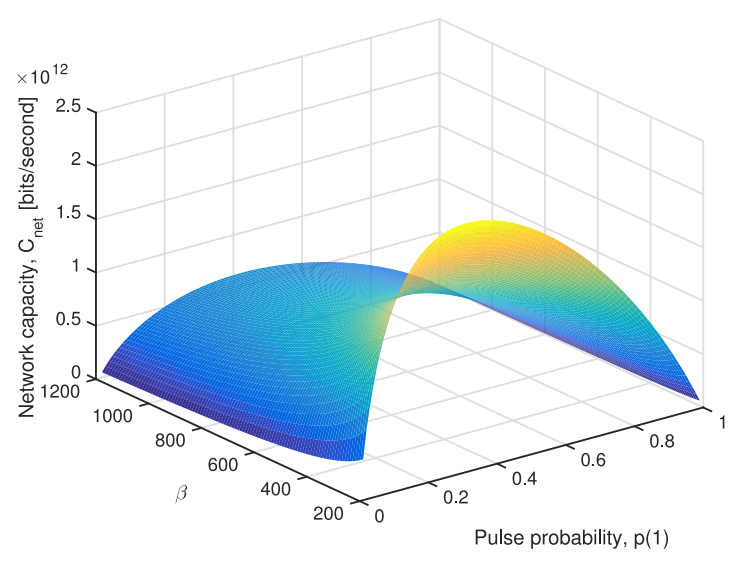

Fig. 7. Network capacity, $C_{\text {net }}$ (where $d=10 \mathrm{~mm}$ and $U=100$ ).

network capacity. One the one hand, the network capacity monotonically decreases with the addition of spreading factor as the pulse probability is fixed. On the other hand, given the identical value of spreading factor, there exists an optimal pulse probability to achieve the maximum network capacity. For example, the network capacity achieves the maximum value $2.34 \times 10^{12}$ bits/second as the best value of pulse probability $p(1)_{\text {best }}$ equals to 0.31 and spreading factor is fixed as 200. It is also observed that when the spreading factor increases from 200 to 1200 (greater than $\beta_{\min }$ ), the corresponding most appropriate pulse probability is changed from 0.31 to 0.41 to guarantee the maximum network capacity.

\section{CONCLUSION}

In this paper, we comprehensively analyzed the tradeoff between energy harvesting and consumption for perpetual nanonetworks in the THz Band. Firstly, the upper bound of the transmitted pulse amplitude was presented for supporting the energy requirement of transmitting one packet based on the energy harvesting structure, and the lower bound of the pulse amplitude was also presented for guaranteeing the SINR threshold. In detail, the mathematical expressions of SINR and interference distribution in the $\mathrm{THz}$ Band were explored on the basis of TS-OOK scheme. Secondly, the minimum spreading factor for perpetual nanonetworks was derived by manipulating the relationship between the energy consumption rate and energy harvesting rate. Finally, given the above obtained parameter constraints, we presented the mathematical model of the maximization of network capacity. Through the integrated optimization of the corresponding system parameters, the maximum network capacity can be achieved.

The simulation results demonstrated that short transmission distance and small spreading factor are recommended to improve the network capacity. In detail, with the identical energy harvesting performance, the minimum spreading factor is dominated by the transmitted pulse energy. The adopted value for spreading factor is suggested to be greater than 200 . The network capacity decreases with the addition of spreading factor, while a big spreading factor contributes to relax the energy requirement. The best pulse probability to achieve the maximum network capacity is dependent on the transmission 
distance and spreading factor. When the transmission distance between two nodes increases from $0.1 \mathrm{~mm}$ to $1 \mathrm{~m}$, the most appropriate pulse probability is from 0.31 to 0.46 as the value of spreading factor belongs to the range from 200 to 1200 .

\section{REFERENCES}

[1] I. F. Akyildiz and J. M. Jornet, "The Internet of nano-things," IEEE Wireless Commun., vol. 17, no. 6, pp. 58-63, Dec. 2010.

[2] I. F. Akyildiz and J. M. Jornet, "Electromagnetic wireless nanosensor networks," Nano Commun. Netw., vol. 1, no. 1, pp. 3-19, Mar. 2010

[3] D. Krishnaswamy, A. Helmy, and D. Wentzloff, "Applications of nanontechnologies in communications," IEEE Commun. Mag., vol. 48, no. 6, pp. 110-111, Jun. 2010.

[4] N. Farsad, A. W. Eckford, S. Hiyama, and Y. Moritani, "On-chip molecular communication: Analysis and design," IEEE Trans. Nanobiosci., vol. 11, no. 3, pp. 304-314, Sep. 2012.

[5] C. Rose and I. S. Mian, "Signaling with identical tokens: Lower bounds with energy constraints," in Proc. IEEE Int. Symp. Inf. Theory Proc., 2013, pp. 1839-1843.

[6] K. V. Srinivas, R. S. Adve, and A. W. Eckford, "Molecular communication in fluid media: The additive inverse Gaussian noise channel," IEEE Trans. Inf. Theory, vol. 58, no. 7, pp. 4678-4692, Jul. 2012.

[7] I. Llatser et al., "Scattering of terahertz radiation on a graphene-based nano-antenna," in Proc. 4th Int. Workshop Theor. Comput. Nanophoton. TaCoNa Photon., 2011, pp. 144-146.

[8] I. Llatser et al., "Characterization of graphene-based nano-antennas in the terahertz band," in Proc. 6th Eur. Conf. Antennas Propag. (EUCAP), 2012, pp. 194-198.

[9] J. M. Jornet and I. F. Akyildiz, "Graphene-based nano-antennas for electromagnetic nanocommunications in the terahertz band," in Proc. 4th Eur. Conf. Antennas Propag. (EUCAP), Barcelona, Spain, 2010, pp. 1-5.

[10] S. Abadal et al., "Wireless nanosensor networks using graphene-based nano-antennas," in Proc. GRAPHENE, Bilbao, Spain, 2011, pp. 1-2.

[11] J. M. Jornet and I. F. Akyildiz, "Channel capacity of electromagnetic nanonetworks in the terahertz band," in Proc. IEEE Int. Conf. Commun. (ICC), 2010, pp. 1-6.

[12] J. M. Jornet and I. F. Akyildiz, "Information capacity of pulse-based wireless nanosensor networks," in Proc. 8th Annu. IEEE Commun. Soc. Conf. Sensor Mesh Ad Hoc Commun. Netw. (SECON), Salt Lake City, UT, USA, 2011, pp. 80-88.

[13] J. C. Pujol, J. M. Jornet, and J. S. Pareta, "PHLAME: A physical layer aware MAC protocol for electromagnetic nanonetworks," in Proc. IEEE Conf. Comput. Commun. Workshops (INFOCOM WKSHPS), Shanghai, China, 2011, pp. 431-436.

[14] R. G. Cid-Fuentes, J. M. Jornet, I. F. Akyildiz, and E. Alarcon, "A receiver architecture for pulse-based electromagnetic nanonetworks in the terahertz band," in Proc. IEEE Int. Conf. Commun. (ICC), Ottawa, ON, Canada, 2012, pp. 4937-4942.

[15] J. M. Jornet, "A joint energy harvesting and consumption model for self-powered nano-devices in nanonetworks," in Proc. IEEE Int. Conf. Commun. (ICC), Ottawa, ON, Canada, 2012, pp. 6151-6156.

[16] R. M. Goody and Y. L. Yung, Atmospheric Radiation: Theoretical Basis. New York, NY, USA: Oxford Univ., 1995.

[17] J. M. Jornet and I. F. Akyildiz, "Channel modeling and capacity analysis for electromagnetic wireless nanonetworks in the terahertz band," IEEE Trans. Wireless Commun., vol. 10, no. 10, pp. 3211-3221, Oct. 2011.

[18] G. Piro, L. A. Grieco, G. Boggia, and P. Camarda, "Nano-Sim: Simulating electromagnetic-based nanonetworks in the network simulator 3," in Proc. 6th Int. ICST Conf. Simulat. Tools Techn., Cannes, France, 2013, pp. 203-210.

[19] J. M. Jornet and I. F. Akyildiz, "Joint energy harvesting and communication analysis for perpetual wireless nanosensor networks in the terahertz band," IEEE Trans. Nanotechnol., vol. 11, no. 3, pp. 570-580, May 2012.

[20] S. Xu, B. J. Hansen, and Z. L. Wang, "Piezoelectric-nanowire-enabled power source for driving wireless microelectronics," Nat. Commun., vol. 1, no. 7, pp. 1-5, Oct. 2010.

[21] Z. L. Wang, "Towards self-powered nanosystems: From nanogenerators to nanopiezotronics," Adv. Funct. Mater., vol. 18, no. 22, pp. 3553-3567, Nov. 2008.

[22] Z. L. Wang and J. Song, "Piezoelectric nanogenerators based on zinc oxide nanowire arrays," Science, vol. 312, no. 5771, pp. 242-246, Apr. 2006
[23] Y. Hu et al., "Self-powered system with wireless data transmission," Nano Lett., vol. 11, no. 6, pp. 2572-2577, May 2011.

[24] S. J. Roundy, "Energy scavenging for wireless sensor nodes with a focus on vibration to electricity conversion," Ph.D. dissertation, Dept. Mech. Eng., Univ. California, Berkeley, CA, USA, 2003.

[25] S. P. Beeby, M. J. Tudor, and N. M. White, "Energy harvesting vibration sources for microsystems applications," Meas. Sci. Technol., vol. 17, no. 12, pp. 175-195, Oct. 2006.

[26] Z. L. Wang, "Energy harvesting for self-powered nanosystems," Nano Res., vol. 1, no. 1, pp. 1-8, Jul. 2008.

[27] P. Wang, J. M. Jornet, M. G. A. Malik, N. Akkari, and I. F. Akyildiz, "Energy and spectrum-aware MAC protocol for perpetual wireless nanosensor networks in the terahertz band," Ad Hoc Netw., vol. 11, no. 8, pp. 2541-2555, Nov. 2013.

[28] M. Pierohon, J. M. Jornet, N. Akkari, S. Almasri, and I. F. Akyildiz, "A routing framework for energy harvesting wireless nanosensor networks in the terahertz band," Wireless Netw., vol. 20, no. 5, pp. 1169-1183, Nov. 2013.

[29] N. Akkari et al., "Joint physical and link layer error control analysis for nanonetworks in the terahertz band," Wireless Netw., vol. 22, no. 4, pp. 1221-1233, 2015, doi: 10.1007/s11276-015-1024-y.

[30] O. Ozel, K. Tutuncuoglu, S. Ulukus, and A. Yener, "Fundamental limits of energy harvesting communications," IEEE Commun. Mag., vol. 53, no. 4, pp. 126-132, Apr. 2015.

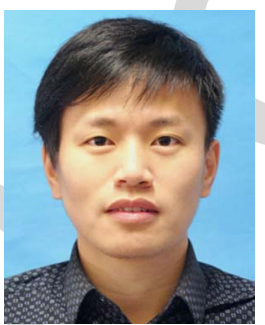

Xin-Wei Yao received the B.S. degree in mechanical engineering and the Ph.D. degree in information engineering from the Zhejiang University of Technology, Hangzhou, China, in 2008 and 2013, respectively. He is currently an Associate Professor with the College of Computer Science and Technology, Zhejiang University of Technology. From 2012 to 2013, he was a Visiting Researcher with Loughborough University, Leicestershire, U.K. From 2015 to 2016, he was a Visiting Professor with the University at Buffalo, State University of New York, Buffalo, NY, USA. His current research interests are in terahertz-band communication networks, electromagnetic nanonetworks, wireless sensor network, and the Internet of Things. He was a recipient of the Distinguished Associate Professor Award and the Outstanding Doctoral Thesis Award at the Zhejiang University of Technology. He is a member of ACM.

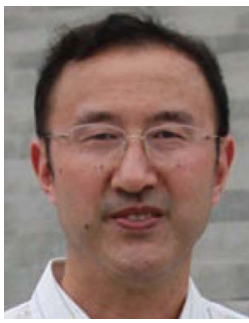

Wan-Liang Wang received the Ph.D. degree in control theory and control engineering from Tongj University, Shanghai, China, in 2001. He is currently a Full Professor with the Zhejiang University of Technology, Hangzhou, China. In 2002, he visited the University of Manchester Institute of Science and Technology, and Loughborough University, U.K. In 2007, he also visited the Georgia Institute of Technology, and the University of Michigan, USA. His research interests include computer networks, networked control, Internet of Things, and artificial intelligence. He was a recipient of the National Outstanding Teacher Award in 2008, and the First National Teacher of Ten Thousand Plan Award in 2014.

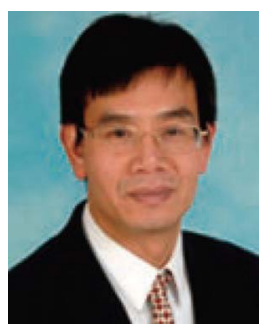

Shuang-Hua Yang (M'05-SM'06) received the B.S. degree in instrument and automation and the M.S. degree in process control from the China University of Petroleum (Huadong), Shandong, China, in 1983 and 1986, respectively, and the Ph.D. degree in intelligent systems from Zhejiang University, Hangzhou, China, in 1991. He is currently a Professor of Networks and Control and Head of Department of Computer Science, Loughborough University, Loughborough, U.K. His current research interests include wireless sensor networks, networked control, safety critical systems, and real-time software maintenance. He is an Editorial Advisory Board Member of the International Journal of Information and Computer Security, and an Associate Editor of the International Journal of Systems Science, the International Journal of Automation and Computing, and the Arabian Journal for Science and Technology. He is a Fellow of IET, a Fellow of InstMC and a Chartered Engineering in U.K 